\title{
Combined airway and oesophageal stenting in malignant airway-oesophageal fistulas: a prospective study
}

\author{
F.J.F. Herth ${ }^{*,+}$, S. Peter ${ }^{\#,+}$, F. Baty ${ }^{\top}$, R. Eberhardt*, J.D. Leuppi ${ }^{\mp}$ and P.N. Chhajed
}

ABSTRACT: Malignant airway-oesophageal fistulas (AEF) are a serious complication of advance oesophageal or lung cancer.

The aim of this study was to assess the quality of life before and after stent insertion, and to examine the role of treatment and location of AEF as factors influencing survival in AEF patients managed with airway and/or oesophageal stent insertion.

112 patients with AEF were included prospectively. $83(74 \%)$ patients had advanced lung cancer and $29(26 \%)$ patients had oesophageal cancers.

Airway stents were inserted in $65(58 \%)$ patients, oesophageal stents in $37(33 \%)$ patients, and both airway and oesophageal stents in 10 (9\%) patients. Seven (6\%) patients developed respiratory failure and required transient ventilator support in the intensive care unit (four patients with airway stenting, two patients with double stents and one patient in the oesophageal stenting group). None of the patients developed stent migration or needed stent repositioning. Overall, mean survival was 236.6 days (airway stent 219.1 days, oesophageal stent 262.8 days and combined airway-oesophageal stent $\mathbf{2 5 2 . 9}$ days). Backward, stepwise regression revealed the site of stent placement (airway and/or oesophagus; $\mathbf{p}<\mathbf{0 . 0 2 8}$ ), exact location of the fistula in airway $(p=0.011)$ and additional treatment with chemotherapy and/or radiation $(p<0.001)$ as independent risk factors predicting increased survival. The mean quality of life score (QoL) was 81 prior to stent insertion and 72 post-stent insertion $(p<0.001)$.

Airway and/or oesophageal stent insertion provides an effective approach to improve the QoL in patients with malignant AEF.

KEYWORDS: Lung cancer, oesophageal cancer, palliative therapy, self-expandable metallic stents, tracheo-oesophageal fistula

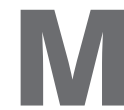
alignant airway-oesophageal fistulas (AEF) are a complication of primary tumour growth or recurrence in oesophageal and lung carcinomas, resulting in a persistent communication between the airways and the upper oesophageal tract. AEF may also occur secondary to radiation or chemotherapy, resulting in tumour dehiscence. This leads to frequent aspiration, with contamination of the airways and poor nutrition. Mortality is a common complication and the mean survival time ranges 1-6 weeks with supportive management alone [1]. Patients with underlying thoracic tumours presenting with symptoms of recent onset of coughing after feeding should raise a high index of suspicion for AEF. A diagnosis is often made using radiological tools, such as barium/gastrograffin oesophagography or computed tomography. Bronchoscopy and oesophagogastroduoedenoscopy (EGD) will help in localising the fistula, and assessing the extent of tumour infiltration and the size of the defect.

Treatments of these fistulas are difficult, considering that most of these patients have an inoperable tumour at the time of diagnosis. Therefore, supportive and palliative treatment forms the basis of improving the quality of life (QoL). Direct surgical fistula closure, bypass or fistula resection do not yield good results, and endoscopic stenting or endoprosthesis are palliative options in this situation [1]. Stents can be placed bronchoscopically in the airways, via EGD in the gus to seal the defect and restore the patency of the passages with resumption of oral feeds [2-5].

Plastic- and silicone-cuffed stents were initially used in the management of AEF [6, 7]. With the use of self-expandable metallic stents (SEMS), there is an increase in the QoL in patients with oesophagus or both in the airways and oesopha-

\section{AFFILIATIONS}

*Pulmonary and Critical Care Medicine, Thoraxklinik, University of Heidelberg, Heidleberg, Germany. Depts of ${ }^{\#}$ Gastroenterology, and "Internal Medicine, University Hospital Basel, Basel, Switzerland. +These authors contributed equally to the study.

\section{CORRESPONDENCE}

P.N. Chhajed

Dept of Pulmonary Medicine University Hospital Basel

Petersgraben 4

CH-4031

Basel

Switzerland

E-mail: pchhajed@uhbs.ch

Received:

March 262009

Accepted after revision:

April 212010

First published online:

June 042010 
inoperable oesophageal and lung tumours [8-10]. A recent study reported improvement of symptoms in $90 \%$ of patients with AEF treated by insertion of oesophageal SEMS [11]. The success rate for closure of AEF using endoscopic methods varies from $87 \%$ to $91 \%$ [11-13].

Data are sparse regarding the benefits of airway or oesophageal stent insertion in the management of malignant AEF. In the endoscopic management of AEF, whether single stent placement in either of the passages is better than double stent placement is not clear. This prospective study was performed to assess the role of self-expandable nitinol airway and/or oesophageal stents in AEF, evaluate the pre- and post-stent insertion QoL, and examine the role of treatment and location of AEF as factors influencing survival in AEF patients managed with airway and/or oesophageal stent insertion.

\section{MATERIALS AND METHODS Patients}

All patients with malignant AEF secondary to oesophageal or lung cancer were prospectively included in the study over a period of 3 yrs. All patients had advanced lung or oesophageal cancer. Based on the symptom profile or site of the primary cancer, patients were treated with bronchoscopic stent insertion in the airways or endoscopic stent insertion in the oesophagus. The decision regarding stent placement was based on the location of the stenosis. If airway stenosis was present, then airway stents were inserted first and, in case of oesophageal stenosis, an oesophageal stent was inserted first. If the fistula was not closed, with the first stent, another stent was implanted directly in the other system. If the fistula was not satisfactorily closed as noted by dye injection with the first stent deployment, then a second stent was inserted immediately in the other system (airway plus oesophageal stent insertion). All procedures were performed under general anaesthesia using flexible video bronchoscopy, rigid bronchoscopy or EGD (Olympus Medical, Tokyo, Japan). Airway and/ or oesophageal Ultraflex nitinol stents (Boston Scientific, Natick, MA, USA) were used for all patients. The dimensions of the stent were chosen based on the site and size of the AEF, and the length of the lesion. Fluoroscopy was routinely used for the placement of oesophageal stents, while airway stents were placed under direct endoscopic visualisation. Informed consent was obtained from all patients and the study was approved by the local ethics committee (University of Heidelberg, Heidelberg, Germany).

\section{Data acquisition}

The baseline study characteristics were recorded, including site of tumour and concurrent treatment with chemotherapy, radiation or surgery. Successful stent deployment, symptomatic relief and complications were also noted. Complete survival follow-up was obtained in all patients.

\section{QoL analysis}

The European Organisation for Research and Treatment of Cancer quality of life questionnaire (EORTC QLQ-C30), which has been developed to assess the QoL of cancer patients [14], was used. The QLQ-C30 incorporates nine multi-item scales: five functional scales (physical, role, cognitive, emotional and social); three symptom scales (fatigue, pain, and nausea and vomiting); and a global health and QoL scale. Several single-item symptom measures are also included in this score. The first QoL questionnaire was administered to all patients prior to stenting. A second, follow-up QoL questionnaire was given to all patients at 6 weeks during follow-up. A high score on a symptom scale or item indicates a high level of problem.

\section{Statistical analysis}

Baseline statistics were compared between the three groups (airway stent only, oesophageal stent only and airway plus oesophageal stents) to the outcomes of complications, survival and QoL scores. All data analyses were computed using the R statistical software [15]. Because all patients died at the time of the analysis, survival times actually correspond to times until death. Survival data are, therefore, uncensored. Survival times are expressed as mean (95\% confidence interval). Betweenclasses comparisons of means were performed using an ANOVA F-test. Factors influencing survival were identified using multiple linear regression. A stepwise, backward elimination was performed in order to remove factors that did not influence survival. The relative importance of each modality of the significant factors was assessed by constrained multiple correspondence analysis. A paired t-test was used to test the significance of the change in questionnaire scores before and after the intervention.

\section{RESULTS}

\section{Demographics}

112 patients (98 males and 14 females) underwent stent insertion in the airways and/or oesophagus secondary to a malignant AEF. 83 (74\%) patients had advanced lung cancers (nonsmall cell lung cancers, $\mathrm{n}=73$; and small-cell lung cancers, $\mathrm{n}=10)$ and $29(26 \%)$ patients had oesophageal nonsmall cell cancer. The median age was 52 yrs (interquartile range 37$81 \mathrm{yrs}) .65$ (58\%) patients underwent airway stent insertion, 37 (33\%) oesophageal stent insertion and 10 (9\%) underwent both airway plus oesophageal stent insertion (table 1). All patients had initial success of fistula closure with the above procedures.

\section{Complications}

Seven $(6 \%)$ patients developed respiratory failure following stent insertion and required mechanical ventilation in the intensive care unit lasting for $<1$ day (four patients with airway stents, one patient with double stents and two with oesophageal stents). None of the patients developed stent migration or required repositioning of the stent. None of the patients had recurrence of AEF until 6 weeks after the initial stenting. During the follow-up period, 24 patients (17 airway, one double and six oesophageal stents) had a recurrence of fistulas accounting for primary failure of stent placement or ineffective closure of AEF, requiring a second stent insertion. There were no complications of perforation or mediastinitis. All patients were followed up and died due to advanced underlying illness.

\section{Survival analysis}

\section{Survival}

All survival times were uncensored. No patients died in the first 30 days after stent insertion. The mean survival (time to death from stent insertion) for the entire group was 236.6 days (95\% CI 221.5-251.6 days). The mean survival was 219.1 days (95\% CI 197.3-240.9 days) for the airway group $(n=65)$, 


\begin{tabular}{|c|c|c|c|c|}
\hline Age yrs mean (range) & $52(37-73)$ & $56(42-71)$ & $57(39-81)$ & $54.3(37-81)$ \\
\hline Males/females & $53 / 12$ & $36 / 1$ & $9 / 1$ & $98 / 14$ \\
\hline \multicolumn{5}{|l|}{ Type of cancer } \\
\hline \multicolumn{5}{|l|}{ NSCLC } \\
\hline Left lung & 25 & 11 & 4 & 40 \\
\hline Right Lung & 17 & 12 & 4 & 33 \\
\hline SCLC & 9 & 0 & 1 & 10 \\
\hline \multicolumn{5}{|l|}{ Localisation of fistula in airway } \\
\hline Trachea & 44 & 5 & 22 & 71 \\
\hline Survival time days median (IQR) & $182(243-279)$ & $249(274-294)$ & $245(278-294)$ & $261(213-293)$ \\
\hline
\end{tabular}

Data are presented as $n(\%)$ or $n$, unless otherwise stated. NSCLC: nonsmall cell lung cancer; SCLC: small cell lung cancer; IQR: interquartile range. ${ }^{\#}$ : airway and oesophageal stenting.

262.8 days (95\% CI 244.4-281.3 days) for the oesophageal group $(\mathrm{n}=37)$; and 252.9 days (95\% CI 192.9-312.9 days) for the double stent group $(n=10)$. Survival was noted to be significantly lower in the group of patients who received only airway stent placement compared to those who received oesophageal or double (airway plus oesophageal) stent insertion (F-test $p=0.023$ ). The survival times based on the treatment and localisation of the fistula are shown in figure 1.

\section{Multivariate analysis}

Specific independent risk factors were analysed for success rates in terms of survival times for all patients. The individual

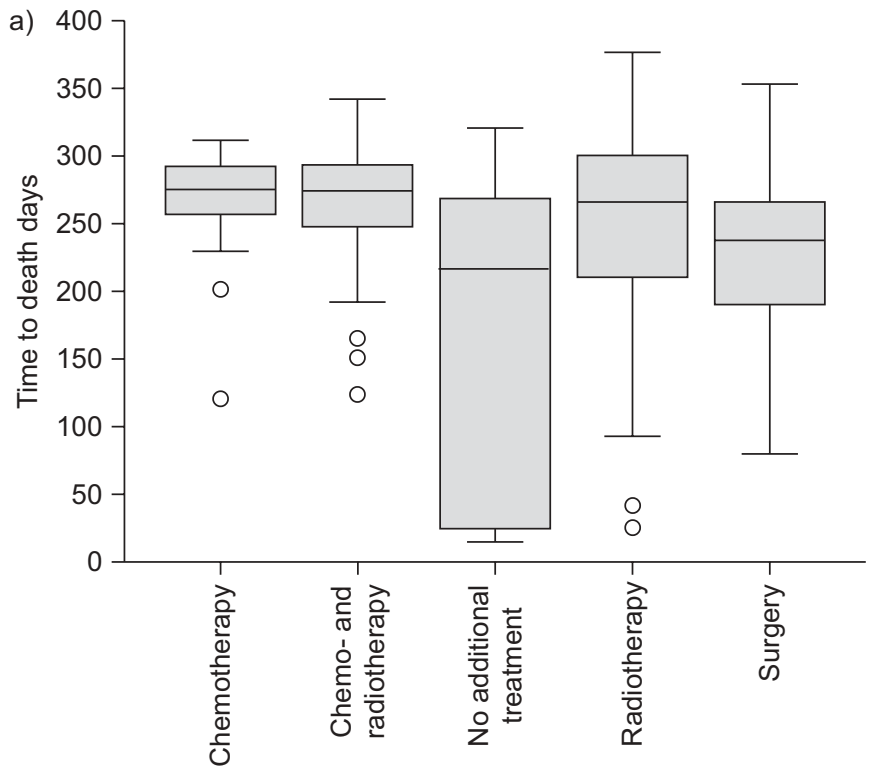

factors analysed in this study were age, sex, localisation of the fistula site in the airway and site of stent placement (airway or oesophageal), treatment with radio- and/or chemotherapy, and complications. The site of stent placement $(\mathrm{p}<0.011)$, localisation of the fistula in the airway $(p=0.023)$ and treatment $(p<0.001)$ were identified as independent risk factors predicting increased or better survival times (fig. 2). For the site of stent placement, oesophageal and airway plus oesophageal sites had a higher survival compared to airway alone. For the localisation of fistula, carina, left main bronchus and trachea had a higher survival than right main bronchus.

b)

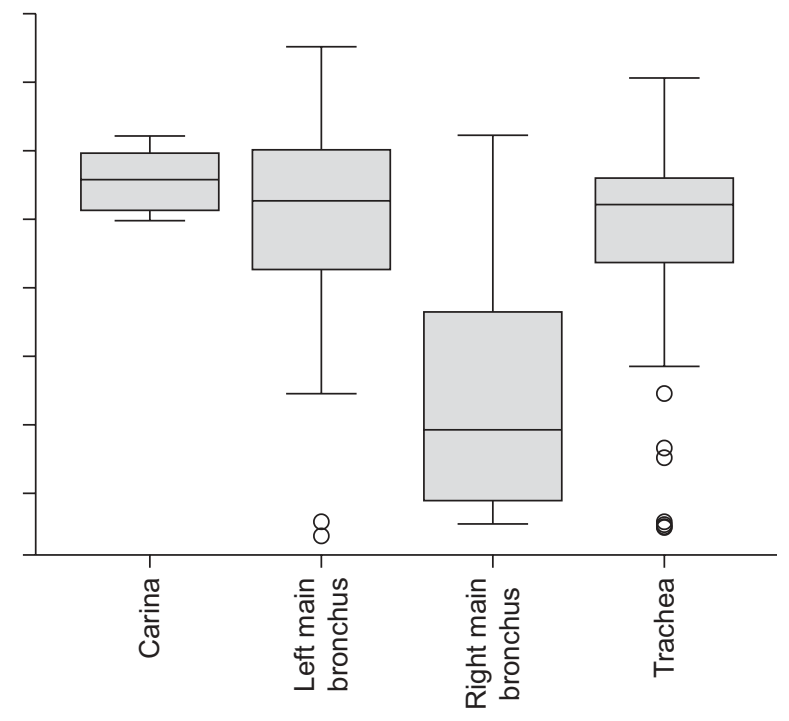

FIGURE 1. Survival analysis with independent factors between stent groups. Box plots showing the relationship between a) survival times and concomitant treatment and b) survival and localisation of the fistula. Boxes represent median and interquartile range (IQR), and whiskers represent \pm 1.5 IQR; 0 : outliers. 


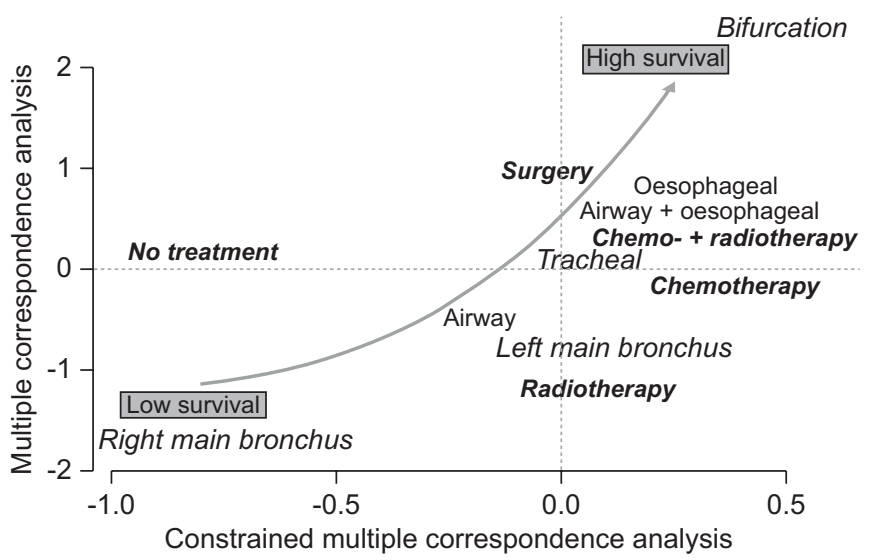

FIGURE 2. Multiple correspondence analyses of factors influencing survival. The $x$-axis displays the factors modalities ordinated by the individuals and after accounting for survival times. The $y$-axis shows how factor modalities are ordinated by individuals without constraints. This mixed plot shows a gradient of survival from the lower left corner (low survival) to the upper right corner (high survival). For example, the combination of oesophageal stent placement, fistula localisation in the carina and surgical post-treatment leads to a relatively better prognosis in terms of survival.

\section{QoL index}

The QoL index (pre- and post-stent insertion) scores are shown in figure 3. The scores were calculated for individual functional and symptoms scores for the oesophageal group, the airway group and the double stenting group. There was significant improvement in QoL scores pre- and post-stent insertion at 6 weeks (fig. 3). There was also marked improvement in the overall health and QoL (paired t-test $\mathrm{p}<0.001$ ).

\section{DISCUSSION}

Malignant AEF poses as a serious complication in patients with advanced neoplasia involving the oesophagus or the lung. The American College of Chest Physicians guidelines for palliative care in patients with a malignant AEF or broncho-oesophageal fistula recommends stent insertion in both the tracheobronchial tree and the oesophagus for symptomatic relief [16]. However, there is paucity of evidence and the net benefit is moderate in terms of morbidity and mortality in the endoscopic palliative management of malignant AEF. The findings of our study show that endoscopic stenting in AEF improves the overall QoL post-stent insertion and, thereby, has a significant role in the management of malignant AEF.

The goal of stent placement in AEF is to provide a seal between the airway and the oesophagus, thereby permitting enteral feeding and reducing bronchial contamination [17, 18]. Potential complications of stent placement in the oesophagus include perforation when placed in the cervical regions of the oesophagus, respiratory compromise due to compression of major airways and stent migration. Covered SEMS have, nevertheless, an advantage over plastic or silicon stents not only in covering large defects, but also in having lower rates of migration along with adequate maintenance of patency of the passages $[3,19,20]$. We observed a primary success of fistula closure in all patients who underwent stenting either in the airway, oesophagus or both systems. 24 patients developed a recurrence of fistula after a period of 6 weeks due to progressive disease. Appropriate

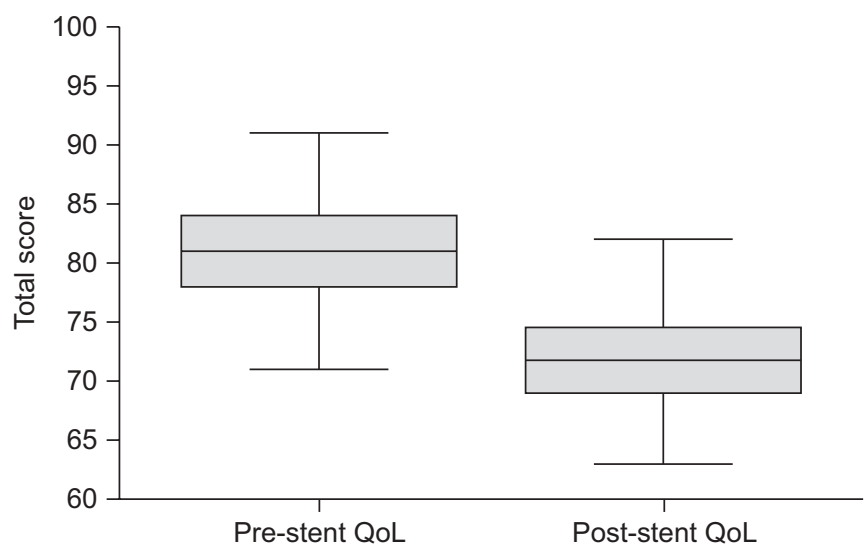

FIGURE 3. Analysis of quality of life (QoL) scores. The box plot shows the mean QoL scores for questions 1-28 pre- and post-stent insertion. Boxes represent median and interquartile range (IQR), and whiskers represent $\pm 1.5 \mathrm{IQR}$.

deployment of a second stent improved the overall success rate and survival in these patients. No major technical difficulties were experienced, supporting the hypothesis that airway and/or oesophageal stent insertion have an important role in the palliation of malignant AEF. Earlier studies have proposed combined airway and oesophageal stenting for malignant AEF [21-23]. The current study was not designed to examine the benefits of combined oesophageal and airway stenting compared to airway or oesophageal stenting in patients with malignant AEF.

The overall mean survival time for the patients in the current study was 236.6 days. The survival times were higher for the patients receiving oesophageal or combined oesophageal and airway stents than patients who received only airway stents. The reason why the airway group performed worse than the other groups is not clear (fig. 2), but it is possible that the airway group had more advanced disease; moreover, patients with stents placed distally (right main bronchus; fig. 1) performed worse compared to those whose stents were placed proximally in the airway (trachea). Among the distally placed airway stents, those stents placed in the right main bronchus had significantly poorer survival than those placed in the left main bronchus. Anatomically, the trachea and left main bronchus have more proximity to the oesophagus compared to the right main bronchus. Hence, an AEF at the level of the right main bronchus would reflect more severe disease, plausibly explaining the lower survival in this group. Similarly, mortality did not seem to be influenced by stent placement, as none of the patients died of stent-related complications. Patients who also received chemoand radiotherapy seem to have a prolonged survival compared to patients who did not receive these therapies.

The overall QoL scores improved significantly after placement of stents (fig. 3). The core QoL was considered appropriate in this study, considering the diverse study population consisting of both oesophageal and lung cancers. The improvement in QoL $<10$ days post-stent insertion was quite evident in most of the patients. There was no significant difference between the groups, demonstrating that airway and/or oesophageal stent insertion was appropriate in improving the overall QoL. Percutaneous endoscopic gastrostomy (PEG) is an alternative procedure that can be considered from a nutritional perspective 
in patients with AEF [24]. To our knowledge, there are no clear guidelines for PEG placement in patients with AEF. The role of PEG was not examined in this study.

In conclusion, airway and/or oesophageal stent insertion provides an effective approach to improve QoL in patients with malignant AEF.

\section{STATEMENT OF INTEREST}

None declared.

\section{REFERENCES}

1 Reed MF, Mathisen DJ. Tracheoesophageal fistula. Chest Surg Clin N Am 2003; 13: 271-289.

2 Tomaselli F, Maier A, Sankin O, et al. Successful endoscopical sealing of malignant esophageotracheal fistulae by using a covered self-expandable stenting system. Eur J Cardiothorac Surg 2001; 20: 734-738.

3 Stemerman DH, Caroline DF, Dabezies M, et al. Nonexpandable silicone esophageal stents for treatment of malignant tracheoesophageal fistulas: complications and radiographic appearances. Abdom Imaging 1997; 22: 14-19.

4 Morgan RA, Ellul JP, Denton ER, et al. Malignant esophageal fistulas and perforations: management with plastic-covered metallic endoprostheses. Radiology 1997; 204: 527-532.

5 Herth F, Becker HD, LoCicero J III, et al. Successful bronchoscopic placement of tracheobronchial stents without fluoroscopy. Chest 2001; 119: 1910-1912.

6 Hegarty MM, Angorn IB, Bryer JV, et al. Palliation of malignant esophago-respiratory fistulae by permanent indwelling prosthetic tube. Ann Surg 1977; 185: 88-91.

7 Hordijk ML, Dees J, van Blankenstein M. The management of malignant esophago-respiratory fistulas with a cuffed prosthesis. Endoscopy 1990; 22: 241-244.

8 Spivak H, Katariya K, Lo AY, et al. Malignant tracheo-esophageal fistula: use of esophageal endoprosthesis. J Surg Oncol 1996; 63: 65-70.

9 Shin JH, Song HY, Ko GY, et al. Esophagorespiratory fistula: longterm results of palliative treatment with covered expandable metallic stents in 61 patients. Radiology 2004; 232: 252-259.

10 Kozarek RA, Raltz S, Marcon N, et al. Use of the $25 \mathrm{~mm}$ flanged esophageal $Z$ stent for malignant dysphagia: a prospective multicenter trial. Gastrointest Endosc 1997; 46: 156-160.
11 Ross WA, Alkassab F, Lynch PM, et al. Evolving role of selfexpanding metal stents in the treatment of malignant dysphagia and fistulas. Gastrointest Endosc 2007; 65: 70-76.

12 May A, Ell C. Palliative treatment of malignant esophagorespiratory fistulas with Gianturco- $Z$ stents. A prospective clinical trial and review of the literature on covered metal stents. Am J Gastroenterol 1998; 93: 532-535.

13 Raijman I, Siddique I, Ajani J, et al. Palliation of malignant dysphagia and fistulae with coated expandable metal stents: experience with 101 patients. Gastrointest Endosc 1998; 48: 172-179.

14 European Organisation for Research and Treatment of Cancer. Quality of Life Questionnaire. http://groups.eortc.be/qol/ Date last updated: July 8, 2010.

15 R Development Core Team. R: A Language and Environment for Statistical Computing. http://cran.r-project.org/doc/manuals/ refman.pdf Date last accessed: 2006. Date last updated: May 31, 2010.

16 Kvale PA, Simoff M, Prakash UB. Lung cancer. Palliative care. Chest 2003; 123: Suppl. 1, 284S-311S.

17 Freitag L, Tekolf E, Steveling H, et al. Management of malignant esophagotracheal fistulas with airway stenting and double stenting. Chest 1996; 110: 1155-1160.

18 Murthy S, Gonzalez-Stawinski GV, Rozas MS, et al. Palliation of malignant aerodigestive fistulae with self-expanding metallic stents. Dis Esophagus 2007; 20: 386-389.

19 Frenken M. Best palliation in esophageal cancer: surgery, stenting, radiation, or what? Dis Esophagus 2001; 14: 120-123.

20 Nomori H, Horio H, Imazu Y, et al. Double stenting for esophageal and tracheobronchial stenoses. Ann Thorac Surg 2000; 70: 1803-1807.

21 Colt HG, Meric B, Dumon JF. Double stents for carcinoma of the esophagus invading the tracheo-bronchial tree. Gastrointest Endosc 1992; 38: 485-489.

22 Alexiou C, Neuhaus H, Kau RJ, et al. Treatment of an esophagorespiratory fistula by insertion of an esophageal Montgomery and tracheal dynamic stent after failure of conventional endoprosthesis. ORL J Otorhinolaryngol Relat Spec 1998; 60: 51-54.

23 van den Bongard $\mathrm{HJ}$, Boot $\mathrm{H}$, Baas $\mathrm{P}$, et al. The role of parallel stent insertion in patients with esophagorespiratory fistulas. Gastrointest Endosc 2002; 55: 110-115.

24 Adler DG, Baron TH, Geels W, et al. Placement of PEG tubes through previously placed self-expanding esophageal metal stents. Gastrointest Endosc 2001; 54: 237-241. 